\title{
The Continuous Shearlet Transform in Arbitrary Space Dimensions
}

\author{
Stephan Dahlke ${ }^{1}$, Gabriele Steidl ${ }^{2}$, and Gerd Teschke ${ }^{3}$ \\ 1 Philipps-Universität Marburg, FB12 Mathematik und Informatik, \\ Hans-Meerwein Straße, Lahnberge, 35032 Marburg, Germany \\ dahlke@mathematik. uni-marburg.de \\ 2 Universität Mannheim, Fakultät für Mathematik und Informatik, \\ Institut für Mathematik, 68131 Mannheim, Germany \\ steidl@math.uni-mannheim.de \\ 3 Hochschule Neubrandenburg - University of Applied Sciences, \\ Institute for Computational Mathematics in Science and Technology, \\ Brodaer Str. 2, 17033 Neubrandenburg, Germany \\ teschke@hs-nb.de
}

\begin{abstract}
This note is concerned with the generalization of the continuous shearlet transform to higher dimensions. Similar to the twodimensional case, our approach is based on translations, anisotropic dilations and specific shear matrices. We show that the associated integral transform again originates from a square-integrable representation of a specific group, the full $n$-variate shearlet group. Moreover, we verify that by applying the coorbit theory, canonical scales of smoothness spaces and associated Banach frames can be derived. We also indicate how our transform can be used to characterize singularities in signals.
\end{abstract}

Keywords. Shearlets, coorbit space theory, square-integrable group representations, Banach frames, detection of singularities.

\section{Introduction}

Modern technology allows for easy creation, transmission and storage of huge amounts of data. Confronted with a flood of data, such as internet traffic, or audio and video applications, nowadays the key problem is to extract the relevant information from these sets. To this end, usually the first step is to decompose the signal with respect to suitable building blocks which are well-suited for the specific application and allow a fast and efficient extraction. In this context, one particular problem which is currently in the center of interest is the analysis of directional information. Due to the bias to the coordinate axes, classical approaches such as, e.g., wavelet or Gabor transforms are clearly not the best choices, and hence new building blocks have to be developed. In recent studies, several approaches have been suggested such as ridgelets [2], curvelets [3], contourlets [7], shearlets [14] and many others. For a general approach see also [13]. Among all these approaches, the shearlet transform stands out because it

Dagstuhl Seminar Proceedings 08482

Structured Decompositions and Efficient Algorithms http://drops.dagstuhl.de/opus/volltexte/2009/1921 
is related to group theory, i.e., this transform can be derived from a squareintegrable representation $\pi: \mathcal{S} \rightarrow \mathcal{U}\left(L_{2}\left(I R^{2}\right)\right)$ of a certain group $\mathcal{S}$, the so-called shearlet group, see [5]. Therefore, in the context of the shearlet transform, all the powerful tools of group representation theory can be exploited.

So far, the shearlet transform is well developed for problems in $I R^{2}$. However, for analyzing higher-dimensional data sets, there is clearly an urgent need for further generalizations. This is exactly the concern of this paper.

To our best knowledge, it seems that there exist only few results in this direction: some important progress has been achieved for the curvelet case in [1] and for surfacelets in [15]. However, for the shearlet approach the question has been completely open.

\section{Multivariate Continuous Shearlet Transform}

In this section, we introduce the shearlet transform on $L_{2}\left(I R^{n}\right)$. This requires the generalization of the two-dimensional parabolic dilation matrix and of the shear matrix, respectively. Let $I_{n}$ denote the $(n, n)$-identity matrix and $0_{n}$, resp. $1_{n}$ the vectors with $n$ entries 0 , resp. 1 . For $a \in I R^{*}:=I R \backslash\{0\}$ and $s \in I R^{n-1}$, we set

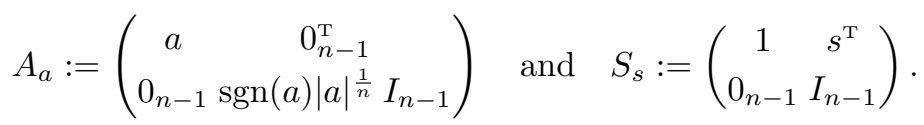

Lemma 1. The set $I R^{*} \times I R^{n-1} \times I R^{n}$ endowed with the operation

$$
(a, s, t) \circ\left(a^{\prime}, s^{\prime}, t^{\prime}\right)=\left(a a^{\prime}, s+|a|^{1-1 / n} s^{\prime}, t+S_{s} A_{a} t^{\prime}\right)
$$

is a locally compact group $\mathcal{S}$ which we call full shearlet group. The left and right Haar measures on $\mathcal{S}$ are given by

$$
d \mu_{l}(a, s, t)=\frac{1}{|a|^{n+1}} d a d s d t \quad \text { and } \quad d \mu_{r}(a, s, t)=\frac{1}{|a|} d a d s d t
$$

In the following, we use only the left Haar measure and use the abbreviation $d \mu=d \mu_{l}$. For $f \in L_{2}\left(I R^{n}\right)$ we define

$$
\pi(a, s, t) f(x)=f_{a, s, t}(x):=|a|^{\frac{1}{2 n}-1} f\left(A_{a}^{-1} S_{s}^{-1}(x-t)\right) .
$$

It is easy to check that $\pi: \mathcal{S} \rightarrow \mathcal{U}\left(L_{2}\left(I R^{n}\right)\right)$ is a mapping from $\mathcal{S}$ into the group $\mathcal{U}\left(L_{2}\left(I R^{n}\right)\right)$ of unitary operators on $L_{2}\left(I R^{n}\right)$. Recall that a unitary representation of a locally compact group $G$ with the left Haar measure $\mu$ on a Hilbert space $\mathcal{H}$ is a homomorphism $\pi$ from $G$ into the group of unitary operators $\mathcal{U}(\mathcal{H})$ on $\mathcal{H}$ which is continuous with respect to the strong operator topology.

Lemma 2. The mapping $\pi$ defined by (1) is a unitary representation of $\mathcal{S}$. 
A nontrivial function $\psi \in L_{2}\left(I R^{n}\right)$ is called admissible, if

$$
\int_{\mathcal{S}}|\langle\psi, \pi(a, s, t) \psi\rangle|^{2} d \mu(a, s, t)<\infty
$$

If $\pi$ is irreducible and there exits at least one admissible function $\psi \in L_{2}\left(I R^{n}\right)$, then $\pi$ is called square integrable. The following result shows that the unitary representation $\pi$ defined in (1) is square integrable.

Theorem 1. A function $\psi \in L_{2}\left(I R^{n}\right)$ is admissible if and only if it fulfills the admissibility condition

$$
C_{\psi}:=\int_{I R^{n}} \frac{|\hat{\psi}(\omega)|^{2}}{\left|\omega_{1}\right|^{n}} d \omega<\infty .
$$

Then, for any $f \in L^{2}\left(I R^{n}\right)$, the following equality holds true:

$$
\int_{\mathcal{S}}\left|\left\langle f, \psi_{a, s, t}\right\rangle\right|^{2} d \mu(a, s, t)=C_{\psi}\|f\|_{L_{2}\left(I R^{n}\right)}^{2} .
$$

In particular, the unitary representation $\pi$ is irreducible and hence square integrable.

\section{Multivariate Shearlet Coorbit Theory}

In this section we want to establish a coorbit theory based on the square integrable representation (1) of the shearlet group. We mainly follow the lines of [4]. For further information on coorbit space theory, the reader is referred to, e.g. [8].

\subsection{Shearlet Coorbit Spaces}

We consider weight functions $w(a, s, t)=w(a, s)$ that are locally integrable with respect to $a$ and $s$, i.e., $w \in L_{1}^{l o c}\left(I^{n}\right)$ and fulfill $w\left((a, s, t) \circ\left(a^{\prime}, s^{\prime}, t^{\prime}\right)\right) \leq$ $w(a, s, t) w\left(a^{\prime}, s^{\prime}, t^{\prime}\right)$ and $w(a, s, t) \geq 1$ for all $(a, s, t),\left(a^{\prime}, s^{\prime}, t^{\prime}\right) \in \mathcal{S}$. For $1 \leq p<$ $\infty$, let

$L_{p, w}(\mathcal{S}):=\left\{F\right.$ measurable $\left.:\|F\|_{L_{p, w}(\mathcal{S})}:=\left(\int_{\mathcal{S}}|F(g)|^{p} w(a, s, t)^{p} d \mu(a, s, t)\right)^{1 / p}<\infty\right\}$,

and let $L_{\infty, w}$ be defined with the usual modifications. In order to construct the coorbit spaces related to the shearlet group we have to ensure that there exists a function $\psi \in L_{2}\left(I R^{n}\right)$ such that

$$
\mathcal{S H}_{\psi}(\psi)=\langle\psi, \pi(a, s, t) \psi\rangle \in L_{1, w}(\mathcal{S}) .
$$

Fortunately, it turns out that (4) can be satisfied in our setting. 
Theorem 2. Let $\psi$ be a Schwartz function such that $\operatorname{supp} \hat{\psi} \subseteq\left(\left[-a_{1},-a_{0}\right] \cup\right.$ $\left.\left[a_{0}, a_{1}\right]\right) \times Q_{b}$, where $Q_{b}:=\left[-b_{1}, b_{1}\right] \times \cdots \times\left[-b_{n-1}, b_{n-1}\right]$. Then we have that $\mathcal{S H}_{\psi}(\psi) \in L_{1, w}(\mathcal{S})$, i.e.,

$$
\|\langle\psi, \pi(\cdot) \psi\rangle\|_{L_{1, w}(\mathcal{S})}=\int_{\mathcal{S}}\left|\mathcal{S H}_{\psi}(\psi)(a, s, t)\right| w(a, s, t) d \mu(a, s, t)<\infty
$$

For $\psi$ satisfying (4) we can consider the space

$$
\mathcal{H}_{1, w}:=\left\{f \in L_{2}\left(I^{n}\right): \mathcal{S H}_{\psi}(f)=\langle f, \pi(\cdot) \psi\rangle \in L_{1, w}(\mathcal{S})\right\},
$$

with norm $\|f\|_{\mathcal{H}_{1, w}}:=\left\|\mathcal{S H}_{\psi} f\right\|_{L_{1, w}(\mathcal{S})}$ and its anti-dual $\mathcal{H}_{1, w}^{\sim}$, the space of all continuous conjugate-linear functionals on $\mathcal{H}_{1, w}$. The spaces $\mathcal{H}_{1, w}$ and $\mathcal{H}_{1, w}^{\sim}$ are $\pi$-invariant Banach spaces with continuous embeddings $\mathcal{H}_{1, w} \hookrightarrow \mathcal{H} \hookrightarrow \mathcal{H}_{1, w}^{\sim}$, and their definition is independent of the shearlet $\psi$. Then the inner product on $L_{2}\left(I R^{n}\right) \times L_{2}\left(I R^{n}\right)$ extends to a sesquilinear form on $\mathcal{H}_{1, w}^{\sim} \times \mathcal{H}_{1, w}$, therefore for $\psi \in \mathcal{H}_{1, w}$ and $f \in \mathcal{H}_{1, w}^{\sim}$ the extended representation coefficients

$$
\mathcal{S H}_{\psi}(f)(a, s, t):=\langle f, \pi(a, s, t) \psi\rangle_{\mathcal{H}_{1, w}^{\sim} \times \mathcal{H}_{1, w}}
$$

are well-defined. Now, for $1 \leq p \leq \infty$, we define the shearlet coorbit spaces

$$
\mathcal{S C}_{p, w}:=\left\{f \in \mathcal{H}_{1, w}^{\sim}: \mathcal{S H}_{\psi}(f) \in L_{p, w}(\mathcal{S})\right\}
$$

with norms $\|f\|_{\mathcal{S C}_{p, w}}:=\left\|\mathcal{S H}_{\psi} f\right\|_{L_{p, w}(\mathcal{S})}$. It holds that $\mathcal{S C}_{1, w}=\mathcal{H}_{1, w}$ and $\mathcal{S C}_{1,1}=$ $L_{2}\left(I R^{n}\right)$.

\subsection{Shearlet Banach Frames}

The Feichtinger-Gröchenig theory provides us with a machinery to construct atomic decompositions and Banach frames for our shearlet coorbit spaces $\mathcal{S C}_{p, w}$. In a first step, we have to determine, for a compact neighborhood $U$ of $e \in$ $\mathcal{S}$ with non-void interior, so-called $U$-dense sets. A (countable) family $X=$ $\left((a, s, t)_{\lambda}\right)_{\lambda \in \Lambda}$ in $\mathcal{S}$ is said to be $U$-dense if $\cup_{\lambda \in \Lambda}(a, s, t)_{\lambda} U=\mathcal{S}$, and separated if for some compact neighborhood $Q$ of $e$ we have $\left(a_{i}, s_{i}, t_{i}\right) Q \cap\left(a_{j}, s_{j}, t_{j}\right) Q=$ $\emptyset, i \neq j$, and relatively separated if $X$ is a finite union of separated sets.

Lemma 3. Let $U$ be a neighborhood of the identity in $\mathcal{S}$, and let $\alpha>1$ and $\beta, \gamma>0$ be defined such that

$$
\left[\alpha^{\frac{1}{n}-1}, \alpha^{\frac{1}{n}}\right) \times\left[-\frac{\beta}{2}, \frac{\beta}{2}\right)^{n-1} \times\left[-\frac{\gamma}{2}, \frac{\gamma}{2}\right)^{n} \subseteq U .
$$

Then the sequence

$$
\left\{\left(\epsilon \alpha^{j}, \beta \alpha^{j\left(1-\frac{1}{n}\right)} k, S_{\beta \alpha^{j\left(1-\frac{1}{n}\right)} k} A_{\alpha^{j}} \gamma m\right): j \in I Z, k \in I Z^{n-1}, m \in I Z^{n}, \epsilon \in\{-1,1\}\right\}
$$

is $U$-dense and relatively separated. 
Next we define the $U$-oscillation as

$$
\operatorname{osc}_{U}(a, s, t):=\sup _{u \in U}\left|\mathcal{S H}_{\psi}(\psi)(u \circ(a, s, t))-\mathcal{S H}_{\psi}(\psi)(a, s, t)\right| .
$$

Then, the following decomposition theorem, which was proved in a general setting in $[8,9,10,11,12]$, says that discretizing the representation by means of an $U$-dense set produces an atomic decomposition for $\mathcal{S C}_{p, w}$.

Theorem 3. Assume that the irreducible, unitary representation $\pi$ is $w$-integrable and let an appropriately normalized $\psi \in L_{2}\left(I R^{n}\right)$ which fulfills

$$
M\langle\psi, \pi(a, s, t)\rangle:=\sup _{u \in(a, s, t) U}|\langle\psi, \pi(u) \psi\rangle| \in L_{1, w}(\mathcal{S})
$$

be given. Choose a neighborhood $U$ of e so small that

$$
\left\|\operatorname{osc}_{U}\right\|_{L_{1, w}(\mathcal{S})}<1
$$

Then for any $U$-dense and relatively separated set $X=\left((a, s, t)_{\lambda}\right)_{\lambda \in \Lambda}$ the space $\mathcal{S C}_{p, w}$ has the following atomic decomposition: If $f \in \mathcal{S C}_{p, w}$, then

$$
f=\sum_{\lambda \in \Lambda} c_{\lambda}(f) \pi\left((a, s, t)_{\lambda}\right) \psi
$$

where the sequence of coefficients depends linearly on $f$ and satisfies

$$
\left\|\left(c_{\lambda}(f)\right)_{\lambda \in \Lambda}\right\|_{\ell_{p, w}} \leq C\|f\|_{\mathcal{S C}_{p, w}}
$$

with a constant $C$ depending only on $\psi$ and with $\ell_{p, w}$ being defined by

$$
\ell_{p, w}:=\left\{c=\left(c_{\lambda}\right)_{\lambda \in \Lambda}:\|c\|_{\ell_{p, w}}:=\|c w\|_{\ell_{p}}<\infty\right\},
$$

where $w=\left(w\left((a, s, t)_{\lambda}\right)\right)_{\lambda \in \Lambda}$. Conversely, if $\left(c_{\lambda}(f)\right)_{\lambda \in \Lambda} \in \ell_{p, w}$, then $f=\sum_{\lambda \in \Lambda} c_{\lambda} \pi\left((a, s, t)_{\lambda}\right) \psi$ is in $\mathcal{S C}_{p, w}$ and

$$
\|f\|_{\mathcal{S C}_{p, w}} \leq C^{\prime}\left\|\left(c_{\lambda}(f)\right)_{\lambda \in \Lambda}\right\|_{\ell_{p, w}} .
$$

Given such an atomic decomposition, the problem arises under which conditions a function $f$ is completely determined by its moments $\left\langle f, \pi\left((a, s, t)_{\lambda}\right) \psi\right\rangle$ and how $f$ can be reconstructed from these moments. This is answered by the following theorem which establishes the existence of Banach frames.

Theorem 4. Impose the same assumptions as in Theorem 3. Choose a neighborhood $U$ of e such that

$$
\left\|\operatorname{osc}_{U}\right\|_{L_{1, w}(\mathcal{S})}<1 /\left\|\mathcal{S} \mathcal{H}_{\psi}(\psi)\right\|_{L_{1, w}(\mathcal{S})} .
$$

Then, for every $U$-dense and relatively separated family $X=\left((a, s, t)_{\lambda}\right)_{\lambda \in \Lambda}$ in $G$ the set $\left\{\pi\left((a, s, t)_{\lambda}\right) \psi: \lambda \in \Lambda\right\}$ is a Banach frame for $\mathcal{S H}_{p, w}$. This means that 
i) $f \in \mathcal{S C}_{p, w}$ if and only if $\left(\left\langle f, \pi\left((a, s, t)_{\lambda}\right) \psi\right\rangle_{\mathcal{H}_{1, w} \times \mathcal{H}_{1, w}}\right)_{\lambda \in \Lambda} \in \ell_{p, w}$;

ii) there exist two constants $0<D \leq D^{\prime}<\infty$ such that

$$
D\|f\|_{\mathcal{S C}_{p, w}} \leq\left\|\left(\left\langle f, \pi\left((a, s, t)_{\lambda}\right) \psi\right\rangle_{\mathcal{H}_{1, w}^{\sim} \times \mathcal{H}_{1, w}}\right)_{\lambda \in \Lambda}\right\|_{\ell_{p, w}} \leq D^{\prime}\|f\|_{\mathcal{S C}_{p, w}} ;
$$

iii) there exists a bounded, linear reconstruction operator $\mathcal{R}$ from $\ell_{p, w}$ to $\mathcal{S C}_{p, w}$ such that

$\mathcal{R}\left(\left(\left\langle f, \psi\left((a, s, t)_{\lambda}\right) \psi\right\rangle_{\mathcal{H}_{1, w}^{\sim} \times \mathcal{H}_{1, w}}\right)_{\lambda \in \Lambda}\right)=f$.

It can be checked that the conditions (10), (11) and (15) can be satisfied, see [6] for details.

\section{Analysis of Singularities}

In this section, we deal with the decay of the shearlet transform at hyperplane singularities. An $(n-m)$-dimensional hyperplane in $I R^{n}, 1 \leq m \leq n-1$, not containing the $x_{1}$-axis can be written w.l.o.g. as

$$
\underbrace{\left(\begin{array}{c}
x_{1} \\
\vdots \\
x_{m}
\end{array}\right)}_{x_{A}}+P \underbrace{\left(\begin{array}{c}
x_{m+1} \\
\vdots \\
x_{n}
\end{array}\right)}_{x_{E}}=\left(\begin{array}{c}
0 \\
\vdots \\
0
\end{array}\right), \quad P:=\left(\begin{array}{c}
p_{1}^{\mathrm{T}} \\
\vdots \\
p_{m}^{\mathrm{T}}
\end{array}\right) \in I R^{m, n-m} .
$$

Then we obtain for

$$
\nu_{m}:=\delta\left(x_{A}+P x_{E}\right)
$$

with the Delta distribution $\delta$ that

$$
\begin{aligned}
\hat{\nu}_{m}(\omega) & =\int_{I R^{n}} \delta\left(x_{A}+P x_{E}\right) e^{-2 \pi i\left(\left\langle x_{A}, \omega_{A}\right\rangle+\left\langle x_{E}, \omega_{E}\right\rangle\right)} d x \\
& =\int_{I R^{n-m}} e^{-2 \pi i\left(-\left\langle P x_{E}, \omega_{A}\right\rangle+\left\langle x_{E}, \omega_{E}\right\rangle\right)} d x_{E} \\
& =\delta\left(\omega_{E}-P^{\mathrm{T}} \omega_{A}\right) .
\end{aligned}
$$

The following theorem describes the decay of the shearlet transform at hyperplane singularities. We use the notation $\mathcal{S H}_{\psi} f(a, s, t) \sim|a|^{r}$ as $a \rightarrow 0$, if there exist constants $0<c \leq C<\infty$ such that

$$
c|a|^{r} \leq \mathcal{S H}_{\psi} f(a, s, t) \leq C|a|^{r} \quad \text { as } \quad a \rightarrow 0 .
$$

Theorem 5. Let $\psi \in L_{2}\left(I R^{n}\right)$ be a shearlet satisfying $\hat{\psi} \in C^{\infty}\left(I R^{n}\right)$. Assume further that $\hat{\psi}(\omega)=\hat{\psi}_{1}\left(\omega_{1}\right) \hat{\psi}_{2}\left(\tilde{\omega} / \omega_{1}\right)$, where $\operatorname{supp} \hat{\psi}_{1} \in\left[-a_{1},-a_{0}\right] \cup\left[a_{0}, a_{1}\right]$ for some $a_{1}>a_{0} \geq \alpha>0$ and $\operatorname{supp} \hat{\psi}_{2} \in Q_{b}$. If

$\left(s_{m}, \ldots, s_{n-1}\right)=\left(-1, s_{1}, \ldots, s_{m-1}\right) P \quad$ and $\quad\left(t_{1}, \ldots, t_{m}\right)=-\left(t_{m+1}, \ldots, t_{n}\right) P^{T}$, 
then

$$
\mathcal{S} \mathcal{H}_{\psi} \nu_{m}(a, s, t) \sim|a|^{\frac{1-2 m}{2 n}} \quad \text { as } a \rightarrow 0 .
$$

Otherwise, the shearlet transform $\mathcal{S H}_{\psi} \nu_{m}$ decays rapidly as $a \rightarrow 0$.

Similar results can be derived for point singularities, see again [6] for details.

\section{References}

1. L. Borup and M. Nielsen, Frame decomposition of decomposition spaces, J. Fourier Anal. Appl., to appear.

2. E. J. Candès and D. L. Donoho, Ridgelets: a key to higher-dimensional intermittency?, Phil. Trans. R. Soc. Lond. A. 357 (1999), 2495-2509.

3. E. J. Candès and D. L. Donoho, Curvelets - A surprisingly effective nonadaptive representation for objects with edges, in Curves and Surfaces, L. L. Schumaker et al., eds., Vanderbilt University Press, Nashville, TN (1999).

4. S. Dahlke, G. Kutyniok, G. Steidl, and G. Teschke, Shearlet Coorbit Spaces and Associated Banach Frames, Preprint Nr. 2007-5, Philipps-Universitt Marburg, 2007.

5. S. Dahlke, G. Kutyniok, P. Maass, C. Sagiv, H.-G. Stark, and G. Teschke, The uncertainty principle associated with the continuous shearlet transform, Int. J. Wavelets Multiresolut. Inf. Process. 6 (2008), 157-181.

6. S. Dahlke, G. Steidl, and G. Teschke, The continuous shearlet transform in arbitrary space dimensions, Preprint Nr. 2008-7, Philipps-Universität Marburg 2008.

7. M. N. Do and M. Vetterli, The contourlet transform: an efficient directional multiresolution image representation, IEEE Transactions on Image Processing 14(12) (2005), 2091-2106.

8. H. G. Feichtinger and K. Gröchenig, A unified approach to atomic decompositions via integrable group representations, Proc. Conf. "Function Spaces and Applications", Lund 1986, Lecture Notes in Math. 1302 (1988), 52-73.

9. H. G. Feichtinger and K. Gröchenig, Banach spaces related to integrable group representations and their atomic decomposition I, J. Funct. Anal. 86 (1989), 307340.

10. H. G. Feichtinger and K. Gröchenig, Banach spaces related to integrable group representations and their atomic decomposition II, Monatsh. Math. 108 (1989), 129-148.

11. H. G. Feichtinger and K. Gröchenig, Non-orthogonal wavelet and Gabor expansions and group representations, in: Wavelets and Their Applications, M.B. Ruskai et.al. (eds.), Jones and Bartlett, Boston, 1992, 353-376.

12. K. Gröchenig, Describing functions: Atomic decompositions versus frames, Monatsh. Math. 112 (1991), 1-42.

13. K. Guo, W. Lim, D. Labate, G. Weiss, and E. Wilson, Wavelets with composite dilations and their MRA properties. Appl. Comput. Harmon. Anal. 20 (2006), 220-236.

14. K. Guo, G. Kutyniok, and D. Labate, Sparse multidimensional representations using anisotropic dilation und shear operators, in Wavelets und Splines (Athens, GA, 2005), G. Chen und M. J. Lai, eds., Nashboro Press, Nashville, TN (2006), 189-201.

15. Y. Lu and M.N. Do, Multidimensional directional filterbanks and surfacelets IEEE Trans. Image Process. 16 (2007) 918-931. 